\title{
Relation between size of optic disc and thickness of retinal nerve fibre layer in normal subjects
}

\author{
Shigeo Funaki, Motohiro Shirakashi, Haruki Abe
}

\begin{abstract}
Aims-To evaluate the relation between the optic disc size and the thickness of the peripapillary retinal nerve fibre layer (RNFL) in normal Japanese subjects by means of scanning laser polarimetry. Methods-Scanning laser polarimetry was performed in $\mathbf{6 0}$ normal subjects. One eye of each subject was randomly selected for study. Using a scanning laser polarimeter, the integral of RNFL thickness was measured totally and regionally within a circular band located 1.75 disc diameters from the centre of the optic disc. The correlation between the optic disc size and the integral of RNFL thickness was examined.

Results-The optic disc size showed a significant correlation with the integral of RNFL thickness $(R=0.497, p<0.001)$. A significant negative correlation was observed between the optic disc size and the ratio of inferior integral to total integral of RNFL thickness $(R=-0.274, p=0.034)$.

Conclusions-The cross sectional area occupied by the RNF, measured by scanning laser polarimetry increased significantly with an increase in optic disc size while the ratio of inferior to total cross sectional area decreased significantly. These facts should be considered when one evaluates the RNFL thickness in patients with progressive optic neuropathies such as glaucoma.
\end{abstract}

(Br f Ophthalmol 1998;82:1242-1245)

Availability of the scanning laser polarimeter now permits the objective and quantitative measurement of the thickness of the retinal nerve fibre layer (RNFL) in vivo. ${ }^{1-10}$ Several investigators reported that, using this instrument, measurements of RNFL thickness were highly reproducible when analysed by the same operator. ${ }^{24-810}$ We have previously reported the reproducibility of measurements of RNFL thickness with this instrument; the mean coefficient of variation of three measurements was $3.9 \% .^{5}$ In normal subjects, measurements of the thickness of RNFL with this instrument resemble those obtained by histological examination. Measurements of the peripapillary thickness of the RNFL in the superior and inferior quadrants exceeded those in the temporal and nasal quadrants. ${ }^{24}{ }^{10-12}$ The thickness of the RNFL has been shown to decrease with an increase in distance from the margin of the optic disc. ${ }^{211-13}$ However, the thickness of the RNFL varies considerably, even among normal subjects. ${ }^{246710}$
Previous studies of humans and other primates have shown that the optic nerve fibre count, as evaluated histologically, increases linearly with an increase in optic disc size. ${ }^{14}{ }^{15}$ However, another histological study of human eyes could not identify a clear correlation between axon count and area of the scleral canal. ${ }^{16}$ To understand the clinical significance of the thickness of the RNFL in vivo, we needed to determine clinically whether the peripapillary RNFL thickness was dependent on the size of the optic disc in the normal individual. The present study therefore examined the relation between optic disc size and the integral of the peripapillary RNFL thickness in normal subjects by means of scanning laser polarimetry.

\section{Subjects and methods}

A total of 120 normal eyes of 60 Japanese volunteers (31 males and 29 females) were studied. Subjects ranged in age from 23 to 75 years, mean 48.4 (SD 16.2) years. There were 10 subjects in each of six groups classified by age: between 20 and 29, 30 and 39, 40 and 49, 50 and 59, 60 and 69, and 70 and 79 years. Informed consent was obtained from each subject. The eyes studied had a best corrected visual acuity above 20/25, a refractive error between -5 and +5 dioptres of sphere or between -2 and +2 dioptres of cylinder, a normal intraocular pressure $\leqslant 21 \mathrm{~mm} \mathrm{Hg}$, a normal appearance of the optic disc, no significant ocular disease found by routine ophthalmological examination, and reliable measurements of visual field (fixation loss $<20 \%$, false negative and false positive $<25 \%$ ) performed on the central 24-2 or 30-2 program of the Humphrey visual field analyser (AllerganHumphrey, San Leandro, CA, USA). Results of the glaucoma hemifield test did not show outside normal limits in each eye. $p$ Values for mean deviation and pattern standard deviation were not $<0.05$.

The thickness of the RNFL was measured with a scanning laser polarimeter (Nerve Fiber Analyzer, version 2.1.15 alpha, Laser Diagnostic Technologies, San Diego, CA, USA). Its basic principles and technical characteristics have been described extensively. ${ }^{1-3}$ In brief, this instrument consists of a confocal scanning laser ophthalmoscope with a polarisation modulator, a cornea polarisation compensator, and a polarisation detection unit. Measurement of RNFL thickness is based on the assumption that the RNFL possesses birefringent properties that change (retard) the state of polarisation of an illuminating laser beam. This change can be measured by deter- 


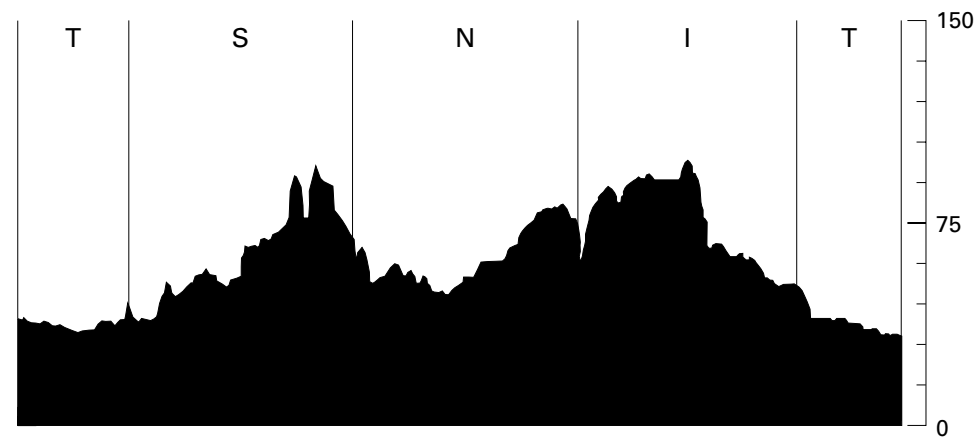

Figure 1 Actual image of the integral of retinal nerve fibre layer RNFL thickness in the nerve fibre analyser. The longitudinal axis indicates the thickness of the RNFL ( $\mu \mathrm{m})$. The integral of RNFL thickness indicates the sectional area which is the integration of the thickness of the RNFL calculated along the transverse axis. $S=$ superior quadrant; $T=$ temporal quadrant; $I=$ inferior quadrant; $N=$ nasal quadrant.

mining the phase shift between extraordinary and ordinary beams. The extent of retardation is linearly correlated with the thickness of the RNFL. Approximately one degree of retardation corresponds to $7.4 \mu \mathrm{m}$ of RNFL thickness measured histologically after removal of the cornea. ${ }^{1}$ The light source consisted of a near infrared diode laser (wavelength $780 \mathrm{~nm}$ ) in which the state of polarisation was modulated. The polarised light penetrates the birefringent RNFL and is partially reflected from the deeper layer of the retina. It is separated from the illuminating light beam by a non-polarising beam splitter. The state of polarisation of the light is analysed by the polarisation detection unit. The electrical signal from the detector is digitised and stored in the memory of a personal computer for later analysis. A total of 65536 retinal locations $(256 \times 256$ pixels $)$ are tested, allowing us to create a retardation map in which the thickness of the RNFL was measured for each retinal location.

In the present study, a 15 degree field size was used and the optic disc was centred in the middle of the image for all image acquisitions. The pupils were not dilated. All measurements were obtained from a mean of three images. The average standard deviation of RNFL thickness in retinal locations (pixels) in the mean image of the three images was within $8 \mu \mathrm{m}$ in each eye. The thickness of the RNFL was measured within a band 10 pixels wide that was located concentrically with the optic disc margin at 1.75 disc diameters. The margin of the optic disc was approximated by an ellipse placed around the inner margin of the peripapillary scleral ring. The integral of the

Table 1 Size of the optic disc and the integral of retinal nerve fibre layer (RNFL) thickness in 60 normal eyes (SD)

Optic disc size $\left(\mathrm{mm}^{2}\right)$

$2.20(0.55)$

Integral of thickness of the RNFL $\left(\mathrm{mm}^{2}\right)$

Total

Superior quadran

Temporal quadrant

Inferior quadrant

Nasal quadrant

$0.544(0.129)$

$0.171(0.045)$

$0.086(0.022)$

$0.174(0.039)$

$0.113(0.035)$

Superior quadrant $v$ temporal quadrant, $\mathrm{p}<0.001$; superior quadrant $v$ inferior quadrant, $\mathrm{p}=0.977$; superior quadrant $v$ nasal quadrant, $\mathrm{p}<0.001$; temporal quadrant $v$ inferior quadrant, $\mathrm{p}<0.001$; temporal quadrant $v$ nasal quadrant, $\mathrm{p}=0.001$; inferior quadrant $v$ nasal quadrant, $\mathrm{p}<0.001$ (ANOVA and Scheffé's test).

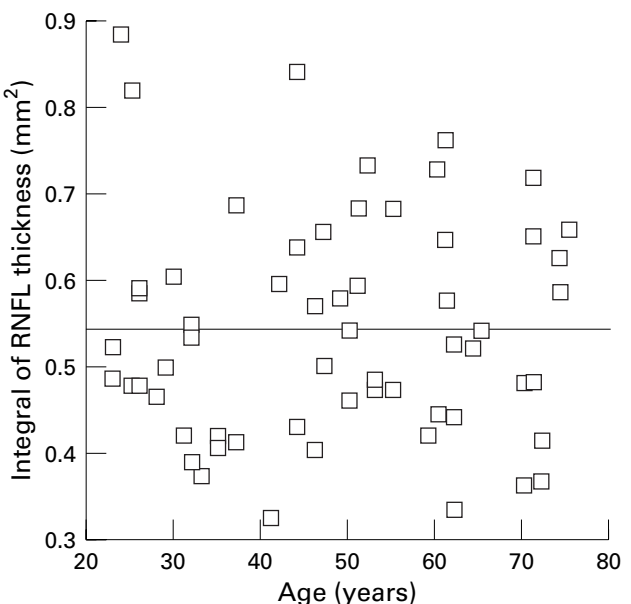

Figure 2 Correlation between age and the integral of thickness of the RNFL in 60 normal eyes $(Y=0.000 x+$ $\left.0.543, R=0.002, R^{2}=0.000, p=0.988\right)$.

thickness of the RNFL was determined within the entire peripapillary circular band and its four 90 degree quadrants (superior, temporal, inferior, and nasal). The actual image of the integral of the thickness of the RNFL was indicated in Figure 1. The long and short radii of the ellipse around the optic disc margin were calculated, and the size of the optic disc was determined by use of the following equation: optic disc area $=\pi \times$ (the long radius of the ellipse $) \times$ (the short radius of the ellipse). To calculate absolute values in the integral of RNFL thickness and optic disc size, errors in magnification were corrected by using Bengtsson's correction with axial length. ${ }^{17}$ Axial length was measured by A scan ultrasonography (Echo Scan US-1600, Nidek, Tokyo, Japan). All analyses were performed by the same operator.

One eye of each of the 60 subjects was chosen at random for analysis. We studied the correlations between age and the integral of thickness of the RNFL, and between the size of the optic disc and the integral of the thickness of the RNFL in 60 eyes.

Data are reported as mean (SD). Linear regression analysis was used to calculate correlation coefficients. Statistical comparisons of groups utilised analysis of variance (ANOVA) followed by multiple comparison test (Scheffé's test). A level of $\mathrm{p}<0.05$ was considered statistically significant.

\section{Results}

The size of the optic disc and the integral of the RNFL thickness in the total peripapillary circular band and its four 90 degree quadrants in 60 normal eyes are shown in Table 1. The integral of the RNFL thickness was greatest in the superior and inferior quadrants, and smallest in the temporal quadrant.

There was no significant correlation between age and the integral of RNFL thickness (Fig $2)$. There was no significant correlation between age and the integral of RNFL thickness in each of the superior, temporal, inferior, and nasal quadrants $\left(R=0.019, R^{2}=0.000, \mathrm{p}=\right.$ $0.885 ; R=-0.054, R^{2}=0.003, \mathrm{p}=0.682 ; R=$ 
Table 2 Correlations between optic disc size $(x)$ and the integral of retinal nerve fibre layer thickness in the superior, temporal, inferior, and nasal quadrants $(Y)$ in 60 normal eyes

\begin{tabular}{lllll}
\hline & Regression line & $R$ & $R^{2}$ & $p$ Value \\
\hline Superior quadrant & $\mathrm{Y}=0.040 \mathrm{x}+0.082$ & 0.494 & 0.244 & $<0.001$ \\
Temporal quadrant & $\mathrm{Y}=0.017 \mathrm{x}+0.049$ & 0.424 & 0.180 & $<0.001$ \\
Inferior quadrant & $\mathrm{Y}=0.029 \mathrm{x}+0.111$ & 0.407 & 0.166 & 0.001 \\
Nasal quadrant & $\mathrm{Y}=0.030 \mathrm{x}+0.046$ & 0.477 & 0.228 & $<0.001$ \\
\hline
\end{tabular}

Table 3 Correlations between optic disc size $(x)$ and the ratio of the superior, temporal, inferior, and nasal quadrants to the total in the integral of retinal nerve fibre layer thickness $(Y)$ in 60 normal eyes

\begin{tabular}{lllll}
\hline Ratio & Regression line & $R$ & $R^{2}$ & $p$ Value \\
\hline Superior quadrant/total & $\mathrm{Y}=0.008 \mathrm{x}+0.296$ & 0.129 & 0.017 & 0.326 \\
Temporal quadrant/total & $\mathrm{Y}=-0.004 \mathrm{x}+0.168$ & -0.083 & 0.007 & 0.528 \\
Inferior quadrant/total & $\mathrm{Y}=-0.014 \mathrm{x}+0.351$ & -0.274 & 0.075 & 0.034 \\
Nasal quadrant/total & $\mathrm{Y}=0.009 \mathrm{x}+0.185$ & 0.222 & 0.049 & 0.088
\end{tabular}

$-0.026, R^{2}=0.001, \mathrm{p}=0.844 ; R=0.047, R^{2}=$ $0.002, \mathrm{p}=0.721$, respectively).

Optic disc size was significantly positively correlated with the integral of RNFL thickness $(R$ $=0.497, R^{2}=0.247, \mathrm{p}<0.001$ ) (Fig 3). A significant correlation was also found between optic disc size and the integral of RNFL thickness in each of the superior, temporal, inferior, and nasal quadrants (Table 2).

Table 3 shows the correlations between optic disc size and the ratio of the superior, temporal, inferior, and nasal quadrants to the total in the integral of RNFL thickness. A significant negative correlation was found between optic disc size and the ratio of the inferior integral to the total integral of RNFL thickness.

\section{Discussion}

It was earlier reported that there was a significant correlation between age and thickness of the RNFL as measured by scanning laser polarimeter-that is, a progressive reduction in thickness of the RNFL with age. ${ }^{24710}$ However, in the present study of normal subjects, we found no significant correlation between age and the integral of RNFL thickness. The age effect in published reports is small, but statistically significant and may not have shown up in our normal subjects.

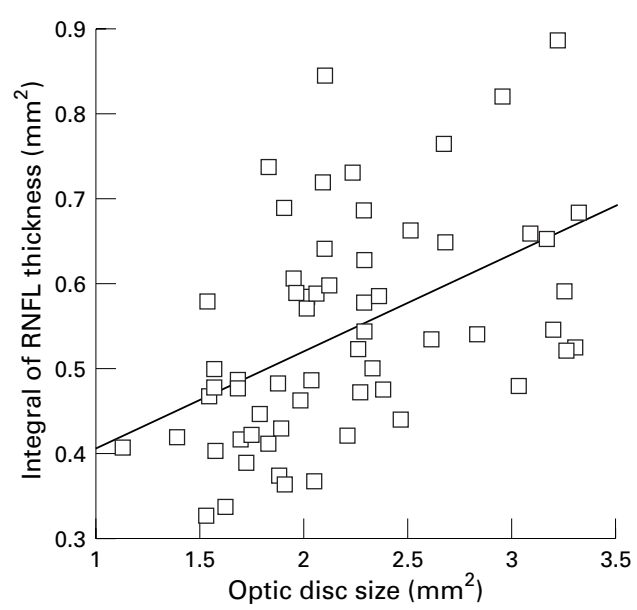

Figure 3 Correlation between size of the optic disc and the integral of thickness of the RNFL in 60 normal eyes $Y=$ $\left.0.116 x+0.288, R=0.497, R^{2}=0.247, p<0.001\right)$.
Although several investigators reported a positive correlation between optic disc size and optic nerve fibre count, as determined histologically in retrobulbar, myelinated optic nerves in non-human primate or human eye $^{14}{ }^{15}$ - that is, optic discs of larger area had more optic nerve fibres than did those of smaller area, other investigators were unable to confirm this correlation. ${ }^{16}$ In the present study of normal humans, there was a significant positive correlation between optic disc size and the total integral of RNFL thickness, as well as the regional integral of RNFL thickness. If the integral of RNFL thickness as measured by scanning laser polarimetry reflects the sum of the sectional area of the nerve fibres present, our results suggest that a larger optic disc has more optic nerve fibres and confirm the previous histological findings. ${ }^{15} 16$

It was previously reported that there was a wide variation among normal individuals in thickness of the RNFL as measured by scanning laser polarimetry, ${ }^{2} 46710$ which may be due to variables in the prenatal regression of retinal ganglion cells in the individual. ${ }^{18}{ }^{19}$ In the present study, a similar wide variation in the integral of RNFL thickness was observed among individuals. There was a considerable overlap in the integral of RNFL thickness among eyes of differing optic disc size.

Although some investigators reported that the size of the optic disc might influence the susceptibility to glaucomatous damage, ${ }^{20}$ others found that the susceptibility to the loss of optic nerve fibre related in glaucoma to be independent of optic disc size. ${ }^{21}$ In the present study, the ratio of the inferior integral to the total integral of RNFL thickness decreased significantly with an increase in optic disc size. This suggests that the cross sectional area occupied by the RNF may not be identical between the superior and inferior quadrants with an increase in optic disc size. Further study is needed to determine whether this optic disc size dependent difference in the ratio of the inferior integral of RNFL thickness is related to a differing susceptibility to glaucomatous damage in patients with differing size of optic disc.

In summary, using scanning laser polarimetry, we demonstrated clinically that the size of the optic disc was significantly correlated with the integral of peripapillary thickness of the RNFL in normal subjects; the cross sectional area occupied by the RNF increased significantly with an increase in optic disc size. The ratio of the inferior integral to the total integral of RNFL thickness differed among eyes of differing optic disc size. Thus, when one evaluates the peripapillary RNFL in patients with progressive optic neuropathies such as glaucoma, one must be aware that the size of the optic disc influences the RNFL data.

The authors have no proprietary interest in any of the materials used in this study.

1 Weinreb RN, Dreher AW, Coleman A, et al. Histopathologic validation of Fourier-ellipsometry measurements of retinal nerve fiber layer thickness. Arch Ophthalmol 1990;108:55760. 
2 Weinreb RN, Shakiba S, Zangwill L. Scanning laser polarimetry to measure the nerve fiber layer of normal and

3 Weinreb RN, Shakiba S, Sample PA, et al. Association between quantitative nerve fiber layer measurement and visual field loss in glaucoma. Am F Ophthalmol 1995;120: $732-8$

4 Chi Q-M, Tomita G, Inazumi K, et al. Evaluation of the effect of aging on the retinal nerve fiber layer thickness using scanning laser polarimetry. f Glaucoma 1995;4:40613.

5 Shirakashi M, Abe H, Sawaguchi S. Relationship between retinal nerve fiber layer thickness and visual field loss in glaucoma.

6 Niessen AGJE, van den Berg TJTP, Langerhorst CT, et al. Retinal nerve fiber layer assessment by scanning laser polarimetry and standardized photography. Am f Ophthal polarimetry and stand

7 Tjon-Fo-Sang MJ, de Vries J, Lemij HG. Measurement by nerve fiber analyzer of retinal nerve fiber layer thickness in normal subjects and patients with ocular hypertension. $\mathrm{Am}$ normal subjects and patients with

F Ophthalmol 1996;122:220-7.
8 Junghardt A, Schmid MK, Schipper I, et al. Reproducibility of the data determined by scanning laser polarimetry. Graefes Arch Clin Exp Ophthalmol 1996;234:628-32.

9 Anton A, Zangwill L, Emdadi A, et al. Nerve fiber layer measurements with scanning laser polarimetry in ocular hypertension. Arch Ophthalmol 1997;115:331-4.

10 Poinoosawmy D, Fontana L, Wu JX, et al. Variation of nerve fibre layer thickness measurements with age and ethnicity by scanning laser polarimetry. $\mathrm{Br} \mathcal{F}$ Ophthalmol 1997;81: $350-4$
11 Radius RL. Thickness of the retinal nerve fiber layer in primate eyes. Arch Ophthalmol 1980;98:1625-9.

12 Quigley HA, Addicks EM. Quantitative studies of retinal nerve fiber layer defects. Arch Ophthalmol 1982;100:80714.

13 Varma R, Skaf M, Barron E. Retinal nerve fiber layer thickness in normal human eyes. Ophthalmology 1996;103: 2114-19.

14 Quigley HA, Coleman AL, Dorman-Pease ME. Larger optic nerve heads have more nerve fibers in normal monkey eyes. Arch Ophthalmol 1991;109:1441-3.

15 Jonas JB, Schmidt AM, Müller-Bergh JA, et al. Human optic nerve fiber count and optic disc size. Invest Ophthalmol Vis Sci 1992;33:2012-18.

16 Mikelberg FS, Yidegiligne HM, White VA, et al. Relation between optic nerve axon number and axon diameter to scleral canal area. Ophthalmology 1991;98:60-3.

17 Bengtsson B, Krakau CE. Correction of optic disc measurements on fundus photographs. Graefes Arch Clin Exp Ophthalmol 1992;230:24-8.

18 Rakic P, Riley KP. Overproduction and elimination of retinal axons in the fetal rhesus monkey. Science 1983;219: 1441-4.

19 Provis JM, van Driel D, Billson FA, et al. Human fetal optic nerve: overproduction and elimination of retinal axons during development. F Comp Neurol 1985;238:92-100.

20 Chi T, Ritch R, Sticker D, et al. Racial differences in optic nerve head parameters. Arch Ophthalmol 1989;107:836-9.

21 Jonas JB, Fernández MC, Naumann GOH. Correlation of the optic disc size to glaucoma susceptibility. Ophthalmology 1991;98:675-80. 